ORIGINAL ARTICLE

\title{
Parental concern and distress about infant pain
}

\author{
L S Franck, S Cox, A Allen, I Winter
}

Arch Dis Child Fetal Neonatal Ed 2004;89:F71-F75

See end of article for authors' affiliations ....................

Correspondence to: Professor Franck, Centre for Nursing and Allied Health Protessions Research, Institute of Child Health, London WCIN 3EH, UK;

Ifranck@ich.ucl.ac.uk

Accepted 22 January 2003

\begin{abstract}
Objective: To describe parent views on infant pain care and to explore relations between parents' experience of their infant's pain care and parental stress.

Design: Descriptive, cross sectional survey.

Setting: Nine neonatal units (196 parents) in the United Kingdom and two neonatal units in the United States (61 parents).

Participants: Parents of preterm and full term infants admitted to hospital.

Interventions: Parents completed a three part questionnaire after the second day of the infant's admission and after they had made at least one previous visit to see their infant in the neonatal unit.

Main outcome measures: Parent concerns about infant pain; parental stress; parent state and trait anxiety. Results: Parents reported that their infants had experienced moderate to severe pain that was greater than they had expected $(p<0.001)$. Few parents $(4 \%)$ received written information, although $58 \%$ reported that they received verbal information about infant pain or pain management. Only $18 \%$ of parents reported that they were shown signs of infant pain, but $55 \%$ were shown how to comfort their infant. Parents had numerous worries about pain and pain treatments. Parental stress was independently predicted by parents' estimation of their infant's worst pain, worries about pain and its treatment, and dissatisfaction with pain information received, after controlling for state anxiety and satisfaction with overall care $\left(F=29.56\right.$, df $\left.6, p<0.001, R^{2}=0.44\right)$. The findings were similar across sites, despite differences in infant characteristics.
\end{abstract}

Conclusions: Parents have unmet information needs about infant pain and wish greater involvement in their infant's pain care. Parent concerns about infant pain may contribute to parental stress.
$\mathrm{P}$ arents of infants receiving medical treatment in the neonatal intensive care unit (NICU) worry that their infant may experience pain, and this concern may contribute to increased parental stress. ${ }^{12}$ Alteration in the parental role, including feeling helpless in not being able to protect the baby and not knowing how to help the baby, was found to be the greatest source of stress for parents of preterm infants during their infant's hospital stay, ${ }^{1}$ and infant suffering was recalled as the worst part of the NICU experience by mothers three years later. ${ }^{3}$ Parents have also shown their high level of concern about infant pain and lack of pain management for infants in the NICU by lobbying medical groups, attracting media attention to the issue, and by pursuing legal action against hospitals and doctors for failing to provide anaesthesia during surgery. ${ }^{4}$

Despite substantial indirect evidence that parents are concerned about infant pain, only one study has specifically examined parental views on pain management in the NICU. In a survey of 95 parents in one United States NICU, Franck and colleagues ${ }^{5}$ found that parents were aware of their infant's pain and that almost half had worries about their infant's pain management. Although most parents (81\%) reported receiving verbal information about infant pain management from staff, few received written information. Parent involvement in pain assessment and management varied, with approximately half of parents reporting that they were shown how to tell if their infant was in pain or how to comfort their infant.

Another recent United States study found that seeing the NICU staff provide comfort to their infant was the most important need of parents, second only to being informed about exactly what was being done to their infant. ${ }^{6}$ Taken together, these findings suggest that there are important gaps in our understanding of parental expectations, involvement, and satisfaction with infant pain management in the NICU setting. Furthermore, little is known about the impact of parental concerns about pain on parental stress and the effectiveness of staff interventions in moderating parental stress related to worry about infant pain. This study used a cross section survey of a large geographically diverse sample to investigate parent views on infant pain care and to explore relations between parents' experience of their infant's pain care and parental stress.

\section{METHODS}

Subjects and procedures

The study was conducted at nine NICUs in the United Kingdom (seven in the London area, one in southeast England, and one in Scotland) and two NICUs in the United States (one West Coast and one East Coast). All parents of infants admitted to the NICU setting were eligible for participation if the parents were English literate. Parents of infants who had been transferred from another neonatal unit were eligible, regardless of the infant's age on admission.

A member of staff (who was not involved in the direct care of the infant) approached the parents and invited them to participate in the study after the second day of the infant's admission, but only if the parent had previously visited their infant at least once. If both parents were present, they were each offered the opportunity to complete the questionnaire independently. Parents were assured that their responses would be confidential and that no individual identities would be used. The Multi-site Research Ethics Committee (United

Abbreviations: CRIB, clinical risk index for babies; NICU, neonatal intensive care unit; PAIN, parent attitudes about infant nociception; PSS, parental stressor scale; STAI, Spielberger state-trait anxiety inventory 
Kingdom sites) and hospital institutional review boards (United States sites) approved the study.

\section{Measures}

Infant age and clinical information such as diagnosis, severity of illness (clinical risk index for babies (CRIB)), surgery, major painful procedures, and analgesia were obtained from the infant's medical record. Parents completed a three part questionnaire consisting of the following instruments.

\section{Parental stressor scale:NICU (PSS:NICU)}

The PSS:NICU ${ }^{1}$ is a 50 item self report scale that measures parental anxiety and stress related to four dimensions of the NICU environment: sights and sounds in the NICU (five items); the infant's appearance (2l items); staff behaviours and communication (13 items); parent interaction with baby (11 items). The parent is asked to rate each item on a five point Likert-type scale from "not experienced" (0) or "not stressful (1)" to "extremely stressful" (5). Mean subscale scores are calculated for each dimension, and the mean total score represents the overall stress level. In studies of parents from North America, the scale showed high test-retest reliability, with correlations ranging from 0.69 for the subscales and 0.87 for the total scale. ${ }^{2}$ Internal consistency has also been shown, with Cronbach's $\alpha$ coefficients ranging from 0.73 to 0.92 for the subscales and from 0.89 to 0.94 for the total scale. ${ }^{128}$

\section{Spielberger state-trait anxiety inventory (STAI)}

The STAI $^{9}$ is a 40 item self report scale that measures trait anxiety (predisposition to be anxious) and state anxiety (response to present situation), showing high concurrent validity with other standard anxiety measures. High testretest reliability ( 0.73 to 0.86 ) is reported for trait anxiety, but low reliability is reported for state anxiety, as would be expected for a response that varies with the situation. ${ }^{9}$ Moderate relations between the PSS:NICU and STAI have been inconsistently found in previous research with North American parents. ${ }^{2}{ }^{8}$ No data have been published on the relation between the PSS:NICU and STAI for parents of infants in neonatal units in the United Kingdom.

\section{Parent attitudes about infant nociception (PAIN)}

The PAIN is a 51 item self report instrument that measures parental views related to parents' experiences with respect to pain and pain management for their infant in the NICU including their expectations (beliefs about future events), involvement (knowledge and participation in care), and satisfaction (personal evaluation of health services and health providers). It is an expanded version of the questionnaire used in a previous study ${ }^{5}$ and is based on previous research that used the American Pain Society's patient pain management satisfaction survey. ${ }^{10}{ }^{11}$ The revised questionnaire (available by request from author) consisted of a combination of yes/no, forced choice, and Likert-type scale questions, with additional space provided for parents to write in comments. In this study, the PAIN questionnaire showed good internal consistency reliability for the expectations and satisfaction dimensions (Cronbach $\alpha=0.71$ and 0.84 respectively).

\section{Data analysis}

Descriptive statistics were calculated for all of the variables of interest (tables 1-6). Parametric and non-parametric tests appropriate for the level of data were used to examine differences in parental, infant, and site characteristics and associations with parental anxiety and NICU related stress. Linear regression analysis was used to quantify the associa-

\begin{tabular}{llll} 
Table 1 & \multicolumn{3}{l}{ Basic infant } \\
& Mean & SD & Range \\
\hline & & & \\
\hline Birth weight (kg) & 2.15 & 1.09 & $0.5-4.8$ \\
Gestational age (weeks) & 33.38 & 5.38 & $23-43$ \\
Postnatal age (days) & 3.62 & 8.93 & $1-72$ \\
Apgar score (5 min) & 8.46 & 1.60 & $0-10$ \\
CRIB score (admission) & 3.59 & 3.72 & $0-16$ \\
\hline CRIB, Clinical risk index for babies. & & \\
\hline
\end{tabular}

tions between parent attitudes about infant pain and NICU related parental stress, after accounting for parent (age, education, state and trait anxiety, delivery mode, visiting) and infant (age, severity of illness, surgical or non-surgical condition, analgesia and sedation) characteristics (table 7).

\section{RESULTS}

Over a 12 month period, 273 parents completed the questionnaires during an NICU visit. About 30\% of eligible parents were not invited to participate because of unavailability of research staff or parents. The refusal rate was $13 \%$. Sixteen participants were dropped from the analysis because the questionnaires were more than $25 \%$ incomplete or because the parents completed one questionnaire together, giving a final sample of 257 respondents.

\section{Parent and infant characteristics}

The sample primarily comprised mothers ( $\mathrm{n}=184)$; however, a large number of fathers $(n=73)$ did participate in the study. In $28 \%$ of the sample, both parents (36 pairs) of an infant completed the questionnaires independently. The responses of mothers and fathers of the same infant were treated as independent in the analyses because each parent was considered to have individual levels of anxiety and views on infant pain. This approach was supported by the fact that responses from the couples did not differ significantly from the responses of the total group or from the respective subgroups of mothers and fathers, nor did they correlate more highly than for the total group. Tables $1-4$ show infant and parent characteristics.

The basic characteristics of the parents did not differ across sites. However, infant clinical characteristics varied by site. In three sites, infants who required surgery were transferred to other hospitals and then transferred back (sometimes the same day) and none of these infants had surgery. In five of the sites, limited surgical procedures were performed-for example, patent ductus arteriosus ligation-and the number of infants receiving surgery in the sample from these sites ranged from one to three. The remaining three sites performed all types of neonatal surgery, and the proportion of surgical infants from these sites ranged from $44 \%$ to $60 \%$. Surgical infants received more opioids and sedatives than non-surgical infants $(\mathrm{p}<0.001)$. Infant severity of illness and parental perception of risk of dying were significantly different across sites $(\mathrm{p}<0.001)$ and showed moderate correlation $(r=0.51, \mathrm{p}=0.001)$.

Parents reported a moderate degree of overall stress, and this was slightly higher for mothers than fathers. The higher maternal stress levels were explained by greater stress related to the sights and sounds of the NICU and to their inability to perform their parental role. Mothers also reported higher levels of state and trait anxiety than fathers. There were no differences in parental stress or state anxiety between sites. Trait anxiety was slightly higher for the United Kingdom parents than the United States parents in the sample (41.71 $(10.93) v 37.23$ (9.70), $\mathrm{p}<0.001)$. However, there was no interaction effect between parent sex and site location (United Kingdom or United States). 


\begin{tabular}{|lll|}
\hline \multicolumn{2}{l}{ Table 2 Clinical details of infants } \\
\hline & No & $\%$ \\
\hline Inborn & 155 & 60 \\
Multiparous birth & 28 & 11 \\
Diagnosis & & \\
Prematurity & 114 & 44 \\
Respiratory & 55 & 21 \\
GI/GU & 26 & 10 \\
Cardiac & 18 & 7 \\
Other & 46 & 18 \\
Mechanical ventilation & 191 & 74 \\
Any supplemental oxygen & 185 & 72 \\
Any vascular access & 248 & 96 \\
Surgery & 54 & 21 \\
Drugs & 148 & 58 \\
Opioids & 65 & 25 \\
Paracetamol & 28 \\
Sedatives & 71 & \\
\hline Gl/GU, Gastrointestinal/genitourinary. & \\
\hline
\end{tabular}

Mothers who had caesarean section deliveries did not see their infants as soon after birth and reported greater stress and state and trait anxiety than mothers who had vaginal deliveries $(\mathrm{p}<0.01)$. Parents who did not hold their infants within 24 hours of birth reported greater stress and also rated their infant as having a greater risk of dying than parents who held their infants sooner $(p<0.01)$.

\section{Parent concerns about infant pain and pain management}

Parents rated their infant's worst pain as moderate to severe on a 0 to 10 scale (mean (SD) $5.47(2.53)$ ). Parents had expected infants to experience less pain (4.18 (2.70); $\mathrm{p}<0.001)$. However, they also expected a high degree of pain relief for their infant.

Table 5 shows parental concerns about infant pain and pain medication. Most parents indicated that they had worries about the effects of pain on their infant, usually that their infants would experience immediate medical problems as a result of pain. Many parents also worried about the long term effects of pain. Few parents expressed concerns over the effects of pain medications. There were no differences between mothers or fathers or among sites on any of the measures of infant pain level or parental concerns about infant pain or pain management.

Parents were generally satisfied with the pain care provided by the neonatal unit staff $(5.20$ (1.01), scale range 1-6). However, there were some differences in expected pain relief and parental satisfaction based on the population served by the neonatal units. Parents in the three sites that had a large proportion of surgical infants expected significantly greater pain relief than parents at the sites that had few or no surgical infants in the study sample (KruskalWallis analysis of variance and Mann-Whitney $U$ post hoc tests, $\mathrm{p}<0.001)$. Parents also reported slightly greater satisfaction with information about pain control and nursing

Table 3 Age and anxiety state of the parents

\begin{tabular}{llll}
\hline & Mothers & Fathers & Total group \\
\hline Age (years) & $31.07(5.77)$ & $33.06(5.47)^{*}$ & $31.62(5.75)$ \\
State anxiety & $51.25(14.10)$ & $46.93(13.92)^{\star *}$ & $49.97(14.16)$ \\
Trait anxiety & $41.61(11.02)$ & $38.25(9.92)^{* *}$ & $40.66(10.81)$ \\
PSS:NICU & $2.38(0.68)$ & $2.18(0.61)^{* *}$ & $2.33(0.67)$ \\
\hline
\end{tabular}

Values are mean (SD).

${ }^{*} p=0.01,{ }^{* *} p=0.03$ compared with mothers.

PSS:NICU, parental stressor scale: neonatal intensive care unit. pain care in the three neonatal units that had a high proportion of surgical infants $(\mathrm{p}<0.001)$.

\section{Information provided to parents about infant pain}

Eleven parents $(4 \%)$ reported that they received written information about infant pain management, whereas 146 $(58 \%)$ reported that they received only verbal information. Parents reported that nurses more commonly provided information about pain than did doctors (41\% $v 28 \%$ ). Parents reported receiving more information about pain in neonatal units that cared for predominantly medical infants $(\mathrm{p}<0.001)$. There were no differences between mothers and fathers in the amount, timing, or type of information that they reported receiving. Parents who reported receiving verbal or written information had more worries about infant pain, had higher expectation of pain relief, were more often shown how to look for signs of pain, and had infants with greater risk of dying ( $\mathrm{p}<0.03-0.001)$.

About a third $(30 \%)$ of parents did not know if their infant received pain medication, and $40 \%$ did not know if the infant received sedatives. Some parents thought that their infant received drugs for pain or sedation when none were documented in the medical record $15 \%$ and $18 \%$ respectively). Fewer parents thought that their infant had not received any analgesia or sedation, when the medical record showed that the infant had received these drugs (3\% and 13\% respectively).

Parents were generally satisfied with the pain information they received (4.48 (1.35), scale range 1-6). Nevertheless, $30 \%(\mathrm{n}=68)$ of the sample wrote in comments indicating that they wish they had received more information about infant pain.

\section{Parent involvement in infant pain care}

Only $18 \%$ of parents reported that they were shown how to identify their infant's signs of pain. However, 55\% reported that they were shown how to comfort their infant. Parents were shown a variety of comfort measures including positioning and non-nutritive sucking (table 6). Most parents stated that they were satisfied with the pain care provided by nurses and doctors. No differences were found in parental stress or satisfaction levels between parents who reported that they were shown infant signs of pain or comfort measures and those who were not.

Few parents reported that they had often been present (12\%) when painful procedures were performed on their infant, whereas $55 \%$ reported that they had sometimes been present, and 33\% reported that they had never been present. Most parents also reported that they were never $(52 \%)$ or not often $(24 \%)$ asked their preference to be present or not when procedures were performed. Nevertheless, more than half of the parents $(57 \%)$ stated that they would prefer to remain at the bedside during procedures. Parents who would prefer to leave the room when procedures were performed on their infant reported greater current pain for their infants and had higher levels of parental stress and state anxiety than did parents who preferred to stay with their infant during procedures $(p<0.03)$. There were no differences between mothers and fathers or among neonatal units in parental involvement in infant pain or parental preferences to be present during procedures. Many parents (87\%) wrote in additional comments about their preferences for greater involvement in their infant's pain care.

\section{Relations between parent concerns about infant pain and parent stress}

Parental stress was unrelated to parental age, ethnicity, or employment status. Parental stress was independently predicted by parents' estimation of their infant's worst pain, 


\begin{tabular}{lll|}
\hline \multicolumn{3}{|c|}{ Table 4 Further characteristics of the parents } \\
\hline & No & $\%$ \\
\hline & 189 & 74 \\
\hline Ethnic background & 35 & 14 \\
White & 14 & 5 \\
Black & 18 & 7 \\
Asian & 161 & 63 \\
Other & 200 & 78 \\
Higher education (above high school or & \\
GCE) & 58 & 23 \\
Employed & 110 & 43 \\
Antenatal diagnosis of neonatal condition & 58 \\
Type of delivery & 79 & 31 \\
Vaginal & 56 & 22 \\
Emergency caesarean & 153 & 60 \\
$\quad$ Elective caesarean & 77 & 30 \\
Saw baby within 4 hours after birth & 86 & 33 \\
Held baby within $24 \mathrm{~h}$ & & \\
NICU visits $>20$ before completing & & \\
questionnaire & & \\
NICU, Neonatal intensive care unit. & & \\
\hline
\end{tabular}

the number of worries that parents had about pain or pain management, and the degree of dissatisfaction with pain information received, after controlling for infant severity of illness and parental state anxiety and satisfaction with overall care $\left(F=29.56\right.$, df $\left.6, \mathrm{p}<0.001, R^{2}=0.44\right)$. Parental state anxiety accounted for $25 \%$ of the variance in parental stress scores, and variables related to parental concerns about infant pain explained a total of $18.2 \%$ of the variance (table 7). Factors that were individually associated with concerns about infant pain or parental stress such as the neonatal unit location, mode of delivery, or length of time before seeing or holding the infant, or whether or not the parent received information about pain did not have a significant effect in predicting parental stress when considered with the other factors in the model.

\section{DISCUSSION}

This is the first study to provide a comprehensive description of parental views on infant pain and pain management in the NICU and to show specific associations between parental concerns about infant pain and parental stress. The degree of parental stress and anxiety reported by parents in this study is similar to previous reports ${ }^{8}$ and, as expected, was related to parental perception of the infant's risk of dying and their ability to see and hold the infant soon after birth. ${ }^{1} 8^{12-14}$ However, it is an important finding that a large proportion of the variance in parental stress was explained by factors relating to parental estimation of infant pain, their worries about infant pain, or their degree of satisfaction with information about infant pain care. The influence of these factors was strikingly consistent despite the diversity of the neonatal units in the sample. The findings from this study are supported by previous research with parents of older children that found that parents have unmet information needs and only passive involvement in pain care for their infant or child. ${ }^{15} 16$

The finding that parents who preferred to be present during painful procedures rated their infants' current pain as less intense and had less anxiety than parents who preferred not to be present is consistent with previous research with older children. ${ }^{17}{ }^{18}$ Parents of sicker infants, those with more worries about pain, and those with higher expectations about pain relief reported receiving more information about infant pain. However, parent self report of receiving pain information and involvement in infants' pain care were not significant predictors of concerns about infant pain, stress, or satisfaction with care. This is inconsistent with the well documented psychological benefits of involving parents in
Table 5 PAIN questionnaire: parent concerns about infant pain and pain management

\begin{tabular}{lll}
\hline & No & $\%$ \\
\hline Worries about infant pain & 69 & 27 \\
None & 131 & 51 \\
Immediate medical problems & 97 & 38 \\
Later medical problems & 73 & 28 \\
Pain memory & 59 & 23 \\
Later pain reaction & 36 & 14 \\
Other worries & 204 & 79 \\
Worries about pain medicine & 22 & 9 \\
None & 20 & 8 \\
Addiction & 13 & 5 \\
Too sleepy & 11 & 4 \\
Breathing problems & 27 & 11 \\
Not enough given & \\
Other worries & \\
\hline PAIN, Parent attitudes about infant nociception. & \\
\hline
\end{tabular}

their infant's general care. ${ }^{19}$ A greater level of parental involvement in infant pain care than shown by the parents in this study, or direct observation of information giving or parental involvement, may be needed to demonstrate the effect of increased active involvement of parents in their infant's pain care on parental stress.

Despite the high incidence of worries about pain and lack of involvement in infant pain care, parents in this study generally reported a high level of satisfaction with all aspects of their infant's pain care. Level of satisfaction was not related to the degree of parents' perception of pain experienced by their infant or to parents' expressed desire for more information and involvement. This finding is consistent with previous studies of adults and children in which there is often a paradox, with high patient satisfaction despite inadequate pain management. ${ }^{11}$

It is unsurprising that parents were more satisfied with pain control information and nursing pain care in the neonatal units that predominantly cared for surgical infants. However, this appears inconsistent with the finding that parents reported receiving more information about infant pain in the units that predominantly cared for medical infants. Perhaps greater emphasis on informing parents about non-pharmacological comfort measures provided to infants to prevent or reduce pain would improve parents' satisfaction with pain care in the non-surgical units. Further research is needed to determine if interventions to improve parental knowledge, involvement, and satisfaction with infant pain care can reduce parental stress. Research is also needed to determine if greater parental understanding of and involvement in their infant's pain care in the NICU setting improves parent-infant attachment and parenting competence or confidence after discharge from the NICU.

Table 6 PAIN questionnaire: parent involvement in infant pain care

\begin{tabular}{lll}
\hline & No & $\% *$ \\
\hline Shown signs of infant pain & 45 & 18 \\
Shown how to comfort infant & 139 & 55 \\
Positioning & 104 & 41 \\
Pacifier & 62 & 25 \\
Patting/rocking & 54 & 21 \\
Swaddling & 53 & 21 \\
Feeding & 38 & 15 \\
Music/toys & 12 & 5 \\
Other methods & 36 & 14 \\
\hline
\end{tabular}

*Percentage based on total number of responses to each question.

PAIN, Parent attitudes about infant nociception. 
Table 7 Predictors of parental stress related to the neonatal intensive care unit

\begin{tabular}{lrlcl}
\hline Variable & \multicolumn{1}{l}{$\boldsymbol{B}$} & $\boldsymbol{R}^{2}$ Change & $\mathbf{9 5 \%} \mathbf{C l}$ & $\mathbf{p}$ Value \\
\hline Parental state anxiety & 0.342 & 0.250 & 0.011 to 0.021 & 0.001 \\
CRIB score & -0.128 & 0.013 & -0.043 to -0.004 & 0.018 \\
Overall satisfaction with care & -0.114 & 0.013 & -0.127 to -0.008 & 0.027 \\
Infant's worst pain level & 0.145 & 0.019 & 0.009 to 0.070 & 0.011 \\
Number of worries about infant pain & 0.307 & 0.110 & 0.083 to 0.181 & 0.001 \\
Satisfaction with information about & -0.175 & 0.053 & -0.140 to -0.034 & 0.001 \\
pain & & & & \\
\hline
\end{tabular}

The findings from this study may be generalisable to other neonatal units because of the diversity of the study sites. However, the findings should be interpreted in the light of the following limitations: parents in the sample were predominantly white, relatively well educated, and English literate; only limited information was obtained from parents so as not to burden them unduly; other factors such as their own previous pain experience or that of their other children may have influenced their views about infant pain; and other socioeconomic factors may have influenced the degree of parental stress. Further research is needed that includes parents from more diverse social and cultural backgrounds and to include other factors that may influence their views of their infant's pain experience or their stress level.

In contemporary management of pain, the family is viewed as playing a key role in the assessment and management of a patient's pain. The lack of information received and involvement in infant pain care reported by parents in this study underscores the gap that exists between professional guidelines $^{20}$ and standards of practice ${ }^{21}$ and the experience of the parents of infants receiving neonatal intensive care. However, this study was not designed to identify the root causes of parents' perceived lack of information and involvement, and we cannot speculate whether information and involvement that parents desire are not being offered or whether parents are not taking up or recalling efforts made by staff. Future research will need to explore the barriers to providing parents with the information and degree of involvement in their infant's pain care that they desire. Nevertheless, the present findings indicate that parents have unmet information needs about infant pain and wish greater involvement in their infant's pain care. Parent concerns about infant pain may contribute to parental stress.

\section{ACKNOWLEDGEMENTS}

This study was supported in part by the Florence Nightingale School of Nursing and Midwifery, King's College London, and the Institute of Child Health and Great Ormond Street Hospital for Children NHS Trust. Research at the Institute of Child Health and Great Ormond Street Hospital for Children NHS Trust also benefits from R\&D funding received from the NHS Executive. The views expressed in this publication are those of the authors and are not necessarily those of the NHS Executive.

We gratefully acknowledge the participating neonatal units for their assistance with this project: Denise Maguire (All Children's Hospital, St Petersburg, FL, USA); Alison Brooks (Alta Bates Hospital, Berkeley, CA, USA); Sarah Keeble, Lyn Ronnie, Dr Dominique Acolet (Chelsea \& Westminster Healthcare NHS Trust, London); Sally Singer, Dr Quen Mok (Great Ormond Street Hospital for Children NHS Trust, London); Yvonne Cousins, Claire Alexander, Dr Grenville Fox (Guy's \& St Thomas' Hospital NHS Trust, London); Dr Yvonne Freer, Dr Neil McIntosh (Lothian University NHS Trust, Edinburgh); Angela Thompson, Dr Jag Ahluwalia, Pauline Fellows, Louise Wells
(Addenbrooks Hospital NHS Trust, Cambridge); Kathy Dunford, Doris Jackman, Dr Sandra Calvert, Jo Bradshaw, Siok Ong (St George's Hospital NHS Trust, London); Jeannie Pompon, Gill Parker (St Mary's Hospital NHS Trust, London); Claire Winter, Dr Gary Hartnoll, Karen Chandler (University Hospital Lewisham NHS Trust, London); Anthea Blake, Denise Stilton, Dr Mark Sellwood (University College London Hospital, London).

\section{Authors' affiliations}

L S Franck, Centre for Nursing and Allied Health Professions Research, Institute of Child Health, London WCIN 3EH, UK

S Cox, School of Nursing and Midwifery, King's College, London, UK A Allen, I Winter, Great Ormond Street Hospital for Children NHS Trust, London, UK

\section{REFERENCES}

1 Miles MS, Funk SG, Carlson J. Parent stressor scale: neonatal intensive care. Nurs Res 1993;42:148-52.

2 Shields-Poe D, Pinelli J. Variables associated with parental stress in neonatal intensive care units. Neonatal Netw 1997;16:29-37.

3 Wereszczak J, Miles MS, Holditch-Davis D. Maternal recall of the neonatal intensive care unit. Neonatal Netw 1997; 16:33-44.

4 Franck LS. The ethical imperative to treat pain in infants: are we doing the best we can? Crit Care Nurse 1997; 17:80-6.

5 Franck LS, Scurr K, Couture S. Parent views of infant pain and pain management in the neonatal intensive care unit. Newborn Infant Nursing Review $2001 ; 1: 106-13$

6 Ward K. Perceived needs of parents of critically ill infants in a neonatal intensive care unit. Pediatr Nurs 2001;27:281-6.

7 The International Neonatal Network. The CRIB (clinical risk index for babies) score: a tool for assessing initial neonatal risk and comparing performance of neonatal intensive care units. Lancet 1993;342:193-8.

8 Miles MS, Funk SG, Kasper M. The stress response of mothers and fathers of preterm infants. Res Nurs Health 1992;15:261-9.

9 Spielberger D. State-trait anxiety inventory (STAI). Palo Alto: Consulting Psychology Press, 1983.

10 Miaskowski C, Nichols R, Brody R, et al. Assessment of patient satisfaction utilizing the American Pain Society's quality assurance standards on acute and cancer-related pain. J Pain Symptom Manage 1994;9:5-11.

11 Ward S, Gordon D. Application of the American Pain Society quality assurance standards. Pain 1994;56:299-306

12 Meyer EC, Garcia Coll CT, Seifer R, et al. Psychological distress in mothers of pretrm infants. J Dev Behav Pediatr 1995;16:412-417.

13 Seideman RY, Watson MA, Corff KE, et al. Parent stress and coping in NICU and PICU. J Pediatr Nurs 1997;12:169-77.

14 Franck S, Bernal H, Gale G. Infant holding policies and practices in neonatal units. Neonatal Netw 2002;21:13-20.

15 Simons J, Franck LS, Robertson E. Parent involvement in children's pain care: views of parents and nurses. J Adv Nurs 2002;36:591-9.

16 Woodgate R, Kristianson $\sqcup$. A young child's pain: how parents and nurses 'take care'. Int J Nurs Stud 1996;33:271-84.

17 Kristensson-Hallstrom I. Strategies for feeling secure influence parents' participation in care. J Clin Nurs 1999;8:586-92.

18 Bauchner H, Vinci R, May A. Teaching parents how to comfort their children during common medical procedures. Arch Dis Child 1994;70:548-50.

19 Klaus MH, Kennell JH. Care of parents. In: Klaus MH, Fanaroff AV, eds. Care of the high-risk neonate, 4th ed. Philadelphia: WB Saunders, 189-211.

20 Royal College of Nursing. Clinical practice guidelines: recognition and assessment of acute pain in children. Bristol: RCN Publishing Co, 1999.

21 Joint Commission on Accreditation of Healthcare Organizations. Pain assessment and management standards. Oakbrook Terrace, IL: JCAHO, 1999. 